\title{
SPRAT: Spectrograph for the Rapid Acquisition of Transients
}

\author{
A. S. Piascik, I. A. Steele, S. D. Bates, C. J. Mottram, R. J. Smith, R.M. Barnsley, B. Bolton \\ Astrophysics Research Institute, Liverpool JMU, ic2 Liverpool Science Park, 146 Brownlow \\ Hill, Liverpool L3 5RF, UK
}

\begin{abstract}
We describe the development of a low cost, low resolution $(R \sim 350)$, high throughput, long slit spectrograph

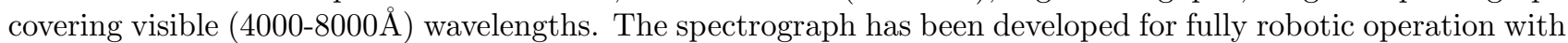
the Liverpool Telescope (La Palma). The primary aim is to provide rapid spectral classification of faint $(V \sim 20)$ transient objects detected by projects such as Gaia, iPTF (intermediate Palomar Transient Factory), LOFAR, and a variety of high energy satellites. The design employs a volume phase holographic (VPH) transmission grating as the dispersive element combined with a prism pair (grism) in a linear optical path. One of two peak spectral sensitivities are selectable by rotating the grism. The VPH and prism combination and entrance slit are deployable, and when removed from the beam allow the collimator/camera pair to re-image the target field onto the detector. This mode of operation provides automatic acquisition of the target onto the slit prior to spectrographic observation through World Coordinate System fitting. The selection and characterisation of optical components to maximise photon throughput is described together with performance predictions.
\end{abstract}

Keywords: robotic telescopes, spectroscopy, spectrographs, transients, astronomical instrumentation

\section{INTRODUCTION}

The Liverpool Telescope (LT) ${ }^{1}$ is a fully robotic 2.0 metre $\mathrm{f} / 10$ telescope at Roque de los Muchachos Observatory on La Palma. Operated by Liverpool John Moores University, it has been at the forefront of time variable and rapid reaction astronomy since 2004. A principal science goal of the LT is the rapid follow up ( $<3$ minutes) of optical transients. ${ }^{2-4}$ The telescope has been optimised for this role with fast slewing, an innovative clamshell dome and the ability to rapidly select between instruments on the Acquisition \& Guidance (A\&G) unit which can host 5 instruments. ${ }^{5}$ The telescope is currently equipped with FRODOSpec, ${ }^{6}$ a medium resolution $(R \sim 2500-5000)$ spectrograph. FRODOSpec uses a small microlens integral field unit (IFU) with a $\sim 10 \times 10$ " field of view to aid robotic (i.e. unsupervised by a human) acquisition. The IFU feeds a bundle of 144 optical fibres of length 25 metres that then present a re-imaged slit at the input to the spectrograph proper, located on the observing floor. The combination of long fibre length, relatively high resolution and other losses in the system means that the sensitivity of FRODOSpec is relatively low. It is therefore best suited to observation of relatively bright $(R<15)$ targets. $^{7}$

A topic of emerging importance in time domain astrophysics is the rapid characterisation of transient phenomena identified in photometric imaging surveys such as Pan-STARRS, ${ }^{8} \mathrm{PTF}^{9}$ and Gaia. ${ }^{10}$ While continuous improvements are being made in classification using light curves alone, ${ }^{11}$ the task is much more straightforward with spectroscopic observations. ${ }^{12}$ These observations need only be at relatively low resolution $(R \sim 100-500)$ in order to make an accurate classification. ${ }^{13}$ SPRAT (Spectrograph for the Rapid Acquisition of Transients) is a project to build a high-throughput, low dispersion $(R \sim 350)$ spectrograph for the LT that is sufficiently stable to operate robotically without the throughput losses associated with an IFU solution. In this paper we describe the spectrograph optical and mechanical design and present the results of bench testing of a prototype. The final instrument is currently in construction and will be deployed to the telescope in summer 2014 .

\section{THE ACQUISITION PROBLEM}

Since the resolution of a spectrograph is determined by the projected width of the input aperture (slit) onto the detector, there is, in general terms, a conflict between the desire for a wide slit (to make acquisition of the target

Further author information: (email a.s.piascik@2013.ljmu.ac.uk) 

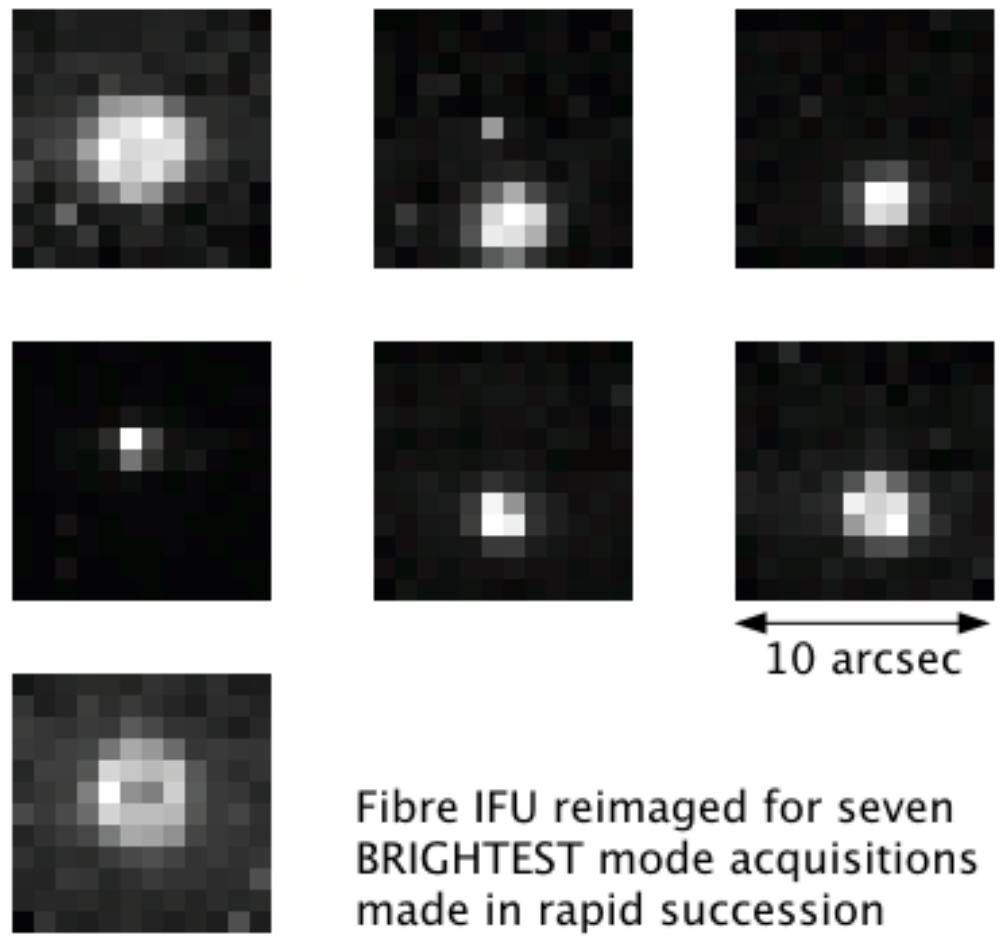

\section{Fibre IFU reimaged for seven \\ BRIGHTEST mode acquisitions made in rapid succession}

Figure 1. Reconstructed FRODOSpec IFU images (collapsed in the wavelength direction) from a series of FRODOSpec acquisitions. The pixel scale is $0.82 \mathrm{arcsec} /$ pixel. The target can be seen to move around the bundle, with an rms of 1.5 arcsec.

onto the entrance aperture straightforward) and a narrow slit (to improve spectral resolution and the rejection of the sky background). For a manually operated telescope, this conflict can generally fairly easily resolved, since a telescope operator may manually adjust the telescope pointing in conjunction with a finder chart. This can often be done while viewing the target and other associated objects in the field using transmitted or reflected light from the slit. For a robotic telescope this " acquisition problem" is more challenging, and can set limits on both the slit width that can be used and the mechanical stability or repeatability of the mechanisms involved.

For our current spectrograph (FRODOSpec) we need only to get the target within a the $\sim 10 \times 10$ arcsec field of view of the IFU. This is still beyond the blind pointing performance of the telescope $(\sim 10 \operatorname{arcsec} \mathrm{rms})$. It is therefore necessary to identify a "magic pixel" on an imaging camera that corresponds to the location a source must be placed such that when the beam is directed onto the IFU it will fall onto it. The location of this magic pixel is assumed to be constant unless either the imaging instrument or IFU is mechanically disturbed, whereupon it must be manually re-derived through a blind search procedure. In normal operation, the process of acquisition is therefore as follows:

1. Point the telescope at the coordinates of the target star and rotate the folding mirror in the A\&G box to direct the beam to an imaging camera (IO:O).

2. Take a 10 second image with IO:O and automatically solve the World Coordinate System (WCS) for the image. ${ }^{14}$

3. Use the WCS solution to derive the location of the target in the IO:O image plane and its offset to the required location in the IO:O image plane that corresponds to the centre of the FRODOSpec IFU (the magic pixel). 
4. If the offset between the magic pixel and the current target location in the focal plane is greater than a given threshold, calculate and apply the required offset to the telescope pointing to move the target to the magic pixel location, and then return to step 2.

5. Once the offset is below the threshold, rotate the folding mirror in the A\&G box to direct the beam onto the IFU, and begin taking spectroscopic data.
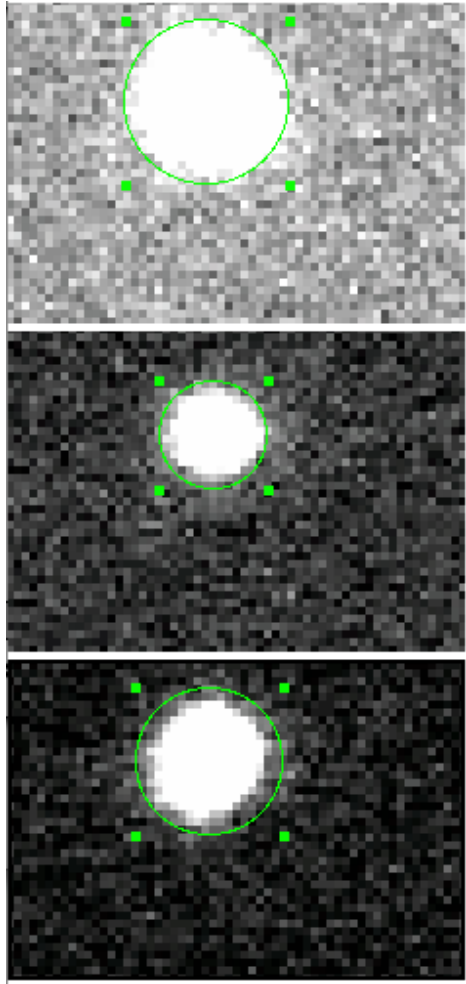
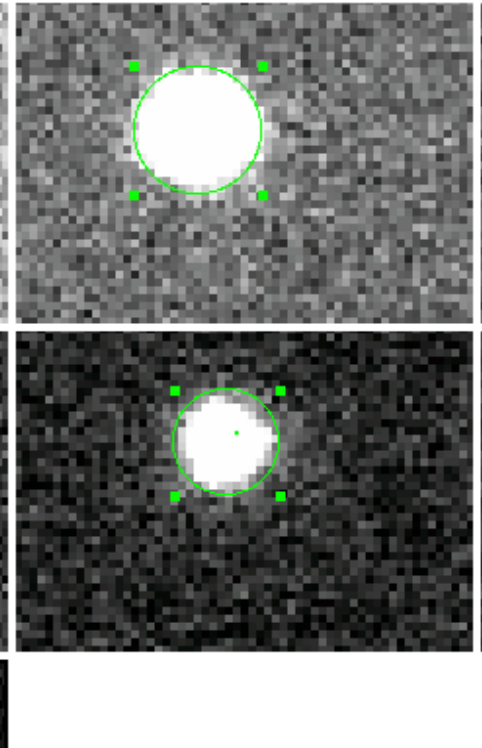
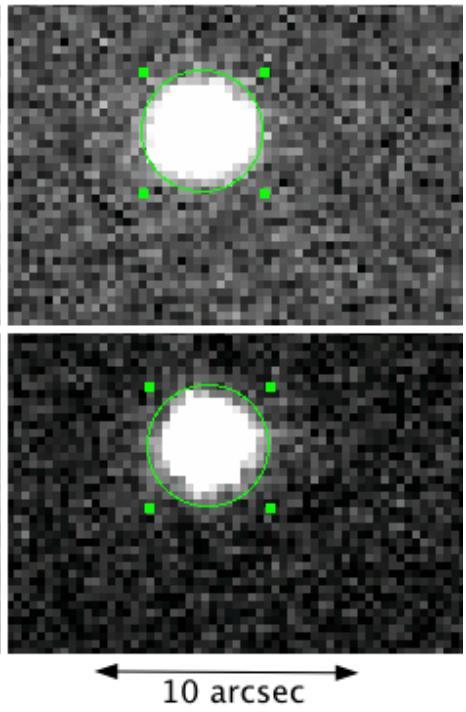

Seven IO:O acquistion images that yield the seven IFU images. These are far more stable and consistent.

Figure 2. Final acquisition images obtained using the IO:O camera for the FRODOSpec IFU images presented in Figure 1. The pixel scale is $0.30 \mathrm{arcsec} /$ pixel. The target can be seen to be more stable, with an rms of 0.6 arcsec.

An example of the results of this procedure can be seen in Figure 1. This shows the reconstructed IFU images from the results of a focus run (hence the PSF of the images varies). The telescope was re-pointed between each image, and the above acquisition procedure performed in full. The offset threshold was set to 1.2 arcsec. The average number of acquisition images taken with this threshold is $\sim 2.1$, implying that most times the initial offset is sufficient and correct, and that no further iteration of the loop is required.

The on-sky rms of the IFU images presented in Figure 1 is $\sim 1.5$ arcsec, compared to the 1.2 arcsec threshold, implying that some additional scatter is probably introduced by the switch between instruments. Evidence for this is presented in Figure 2. This figure presents the final IO:O images taken at the end of each acquisition sequence. The on-sky rms scatter in the location of the target star in these images is $\sim 0.6$ arcsec, implying that the instrument switch is contributing the majority (formally $\sim 1.4$ arcsec) of the IFU image scatter.

In order to further characterise the acquisition problem associated with the telescope pointing and offsets (i.e. ignoring contribution caused by switching instruments switch) we carried out an analysis of one months worth of FRODOSpec acquisitions between 2014 March 10 and 2014 April 10. During this period 74 acquisitions were carried out with the threshold set to 1.2 arcsec. The results of the "self-acquisition" on IO:O are presented in Figure 3. It can be seen that (as required by the algorithm) all of the objects fall within the 1.2 arcsec threshold. Ignoring the stellar PSF and associated slit losses, a minimum slit width of 2.4 arcsec would therefore be necessary to guarantee acquisition using this threshold. With a 2 arcsec slit the fraction acquired would 


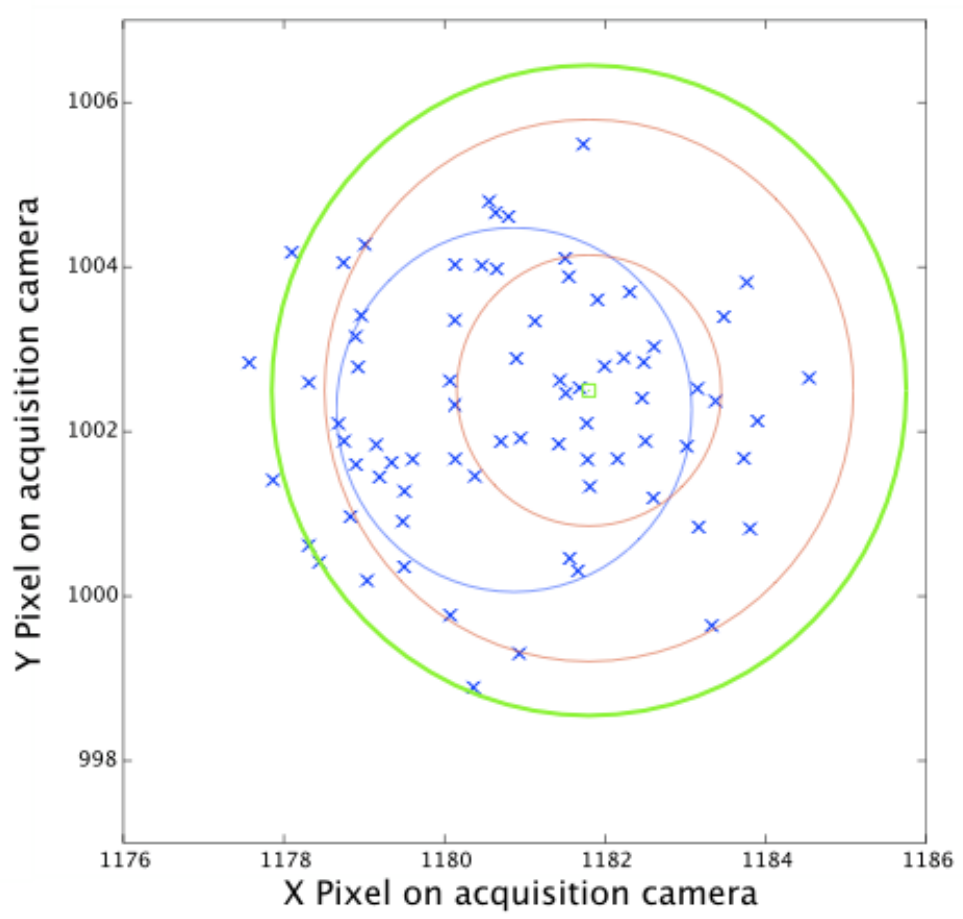

Figure 3. Map of location (blue crosses) in detector focal plane (X and $\mathrm{Y}$ axes show pixel coordinates where 1 pixel $=$ $0.30 \mathrm{arcsec}$ ) of target object in final IO:O acquisition frames. The small green square shows the location of the "magic pixel". The green circle is of radius $1.2 \operatorname{arcsec}$ (the offset threshold), and therefore every cross (within very small limits of error associated with the centroiding algorithm used) falls within it. The blue circle is centered on the mean of the data, and has a radius of 0.67 arcsec corresponding to the rms of the data. The red circles are of radius 0.5 and 1.0 arcsec, and therefore encircle the acquisitions that would have been successful with slits of 1 and 2 arcsec width respectively..

be $\sim 85 \%$ and with a 1.5 arcsec slit this would reduce to $\sim 74 \%$. This experiment was then repeated in the period 2014 April 11 to 2014 April 23 with the acquisition threshold reduced to 1.0 arcsec. During this period 17 acquisitions were attempted, with a mean of 2.9 iterations (maximum 5) required to reach the 1.0 arcsec threshold, implying a maximum slit width of 2.0 arcseconds.

We note that once the object is within the slit and the telescope autoguider is locked, then it should be possible to make sub arcsecond adjustments to the telescope pointing in order to "peak up" the flux in the centre of the slit. Therefore as long as the object can be initially acquired somewhere in the slit width, slit losses due to centering should not be a significant problem.

\section{OPTICAL DESIGN}

The basic equation for the resolution $R$ of a spectrograph operating in first order at wavelength $\lambda$ with a collimated beam width $W$ and slit width $\chi$ on a telescope of primary mirror diameter $D$ is given by:

$$
R=\frac{\rho \lambda W}{\chi D}
$$

where $\rho$ is the grating line density in lines/metre.

As described in Section 2, the acquisition problem for a robotic instrument will drive a number of elements of the design. For SPRAT we identified the following constraints from the measured telescope performance: 
Tranmission Losses

AF Nikkor $85 \mathrm{~mm}$ f/1.8D

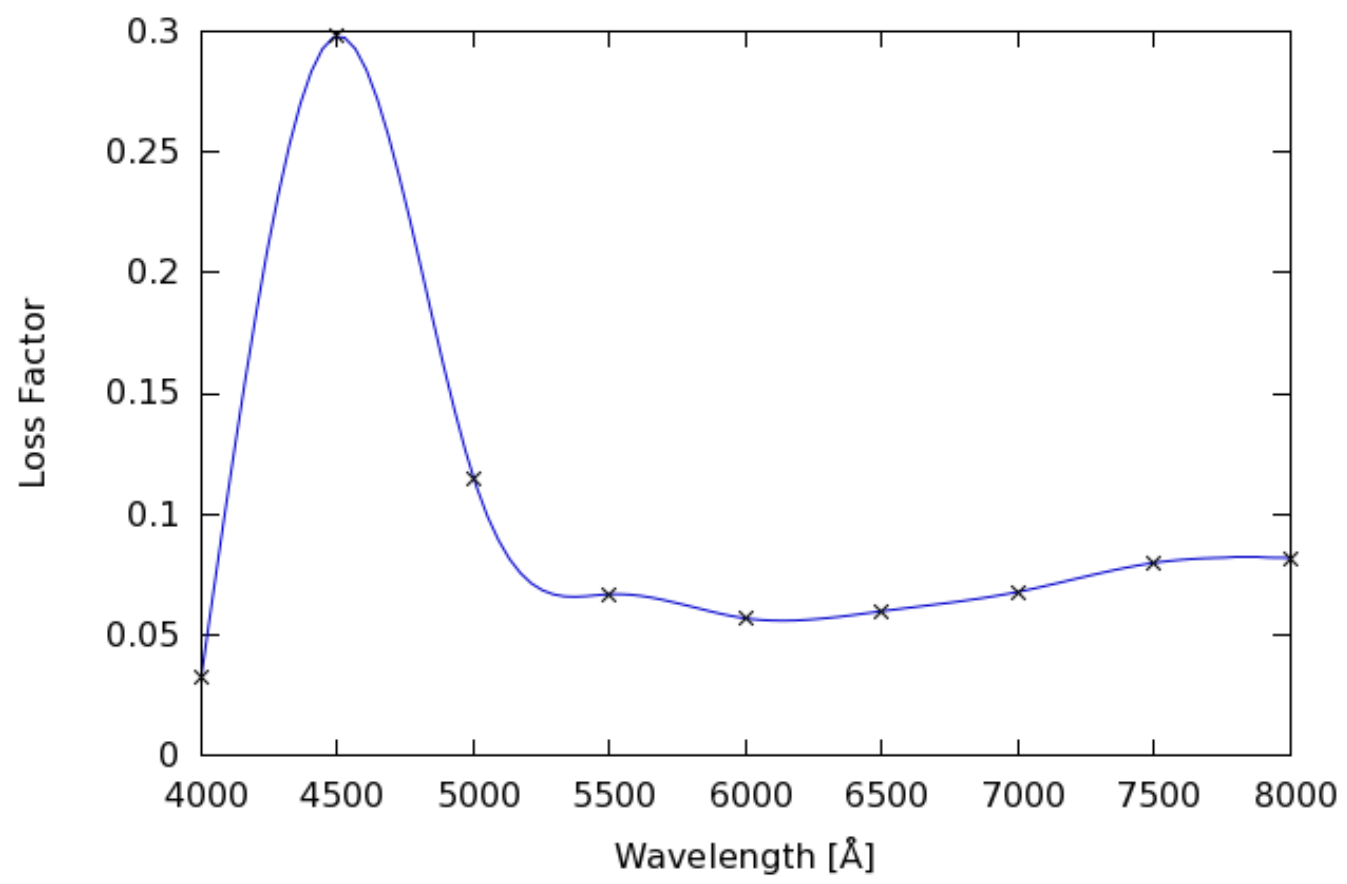

Figure 4. The CCD lens transmission losses

- Slit width in the range 1.5 to 2.5 arcsec. The upper limit is set by the known acquisition performance of the telescope and the lower limit by our aspiration for better spectral resolution should it be improved upon.

- The imaging acquisition mode must be integral to the instrument, otherwise rotation of the A\&G mirror to switch between instruments will dominate the acquisition error budget and force an even wider slit.

Given the uncertainty in optimal slit width, a variable width, symmetrically opening, knife-edge design made by Spiers-Roberston was chosen. The requirements naturally lead to a transmitting spectrograph design with a straight optical path where the slit and dispersive mechanism can be removed from the beam in acquisition, and then reinserted for spectroscopy. It should be noted that a disadvantage of a straight through optical design is its length, both in terms of potential for flexure and the physical space envelope at the telescope A\&G box (which dictated a maximum instrument length of $\sim 740 \mathrm{~mm}$ including the detector assembly.)

In order to obtain a high efficiency, we chose to use a volume phase holographic (VPH) grating. ${ }^{15}$ Commercial versions of these gratings are now available at low cost off the shelf with grating densities between 600 and 1800 $\mathrm{l} / \mathrm{mm}$ and diameters of 25 and $50 \mathrm{~mm}$. By sandwiching the grating between two small angle prisms, a straight through optical arrangement can be devised ${ }^{15}$ which inputs the beam at the optimal angle (equivalent to a "blaze" angle in a conventional grating) for maximum throughput. We chose to use a $600 \mathrm{l} / \mathrm{mm}$ grating of diameter $25 \mathrm{~mm}$. This has a required input angle of $10.2^{\circ}$, and the grating equation shows that at first order it will have a similar output angle of $10.2^{\circ}$ at $6000 \AA$. We therefore sandwiched the grating between two wedge prisms of angle $10.0^{\circ}$.

Since gratings require operation in a collimated beam, this gives the opportunity to adjust the image scale at the detector as compared to the focal plane by suitable choice of focal length of collimating and camera lenses. There are two basic constraints that must be satisfied in arriving at the eventual choice of these values - overall field of view in imaging mode (to ensure sufficient objects are observed that a WCS fit may be made to allow 
automated acquisition) and pixel scale (to ensure that the re-imaged slit is optimally sampled on the detector). Considering the field of view requirement for acquisition, our experience with FRODOSpec and its operation with our previous imaging camera RATCam showed that with a field of view of 21 square arcminutes, successful acquisition is possible in $>94 \%$ of cases.

As a detector we chose the Andor iDus 420 BV camera with 1024x255 pixels, to be aligned with the wavelength direction running along the long axis. Each pixel is $26 \times 26 \mu \mathrm{m}$. The camera has a quantum efficiency (QE) $>70 \%$ over most of the 4000-8000 $\AA$ range and exhibits a relatively low readout noise (9 $\mathrm{e}^{-}$max.) and low dark current $\left(\sim 0.01 \mathrm{e}^{-}\right.$pixel $\left.^{-1} \mathrm{sec}^{-1}\right)$ at $-60^{\circ} \mathrm{C}$.

For the LT (with final focal length $20 \mathrm{~m}$ ) this detector would give an image size on the sky of $\sim 4.6 \times 1.1$ arcminutes (i.e. $\sim 5$ square arcmin) with a pixel scale of 0.27 arcsec/pixel in direct imaging. This pixel scale is rather small compared to the median telescope image quality of $\sim 0.9 \operatorname{arcsec}$ and the area of sky imaged too small for reliable WCS acquisition. A $\sim 2: 1$ imaging system is therefore required to increase the pixel scale to the optimal $\sim 0.5 \mathrm{arcsec} /$ pixel and $\sim 20$ square arcmin field of view. After considering a variety of trade offs, we selected an optical system with collimator focal length $150 \mathrm{~mm}$ and camera focal length $85 \mathrm{~mm}$. This yields a beam width $W=15 \mathrm{~mm}$ and hence resolution with a $1.5 \operatorname{arcsec}$ slit (Equation 1 ) of $R \sim 350$. The linear dispersion at the detector will be 0.2 Angstroms per micron, i.e. $\sim 5$ Angstroms per pixel. A spectral range from 4000 to $8000 \AA$ can therefore be accommodated on the detector over $\sim 800$ pixels, and the spectral sampling will be close to optimal with $\sim 2.5$ pixels per resolution element. The slit height is $27.5 \mathrm{~mm}$ stopped down to $9 \mathrm{~mm}$ at the exit to produce $\sim 200$ pixel high aperture image centered on the CCD $(\sim 1.5 \operatorname{arcmin}$ on the sky). The unilluminated areas provide a measure of the bias and dark level in the images.

It is important to match the optical design of the instrument to the telescope it will be used with. ${ }^{16}$ Ideally the exit pupil of the telescope will be coincident and co-axial with the grating of the spectrograph. In addition space constrains at the A\&G box constrain the overall instrument length to $<740 \mathrm{~mm}$ (preferably $<720 \mathrm{~mm}$ to avoid using minimum cable radii). To achieve this an achromatic doublet field lens, $(\mathrm{D}=50 \mathrm{~mm}, \mathrm{f}=200 \mathrm{~mm})$ was incorporated into the design immediately following the slit. The field lens mount incorporates a long-pass filter

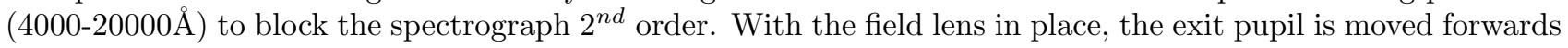
to $48.6 \mathrm{~mm}$ after the collimator lens, and the final instrument length is $645 \mathrm{~mm}$.

For the collimator lens we chose an achromatic doublet, $(\mathrm{D}=50 \mathrm{~mm}, \mathrm{f}=150 \mathrm{~mm})$ with a VIS-NIR anti-reflection coating. VIS-NIR has $<1.0 \%$ reflection loss between $4000-10000 \AA$ for normally incident light. The camera imaging lens chosen was a Nikon AF Nikkor 85mm f/1.8D 6 element photographic lens set to maximum aperture and $\infty$ focus verified by imaging a distant $(>2 \mathrm{~km})$ object. As no published transmission information was available, we made measurements using a Hilger \& Watts monochromator source and the CCD camera. A lossless pin-hole lens allowed the monochromator output to be imaged onto the CCD even when the Nikon lens was absent and the attenuation was measured by comparing the flux at several wavelengths with and without the lens present. The results of this are presented in Figure 4.

\section{EFFECT OF GRATING TILT}

It is known that tilting a VPH grating to adjust the angle of the input beam will shift the wavelength of maximum throughput. ${ }^{17}$ The effect of varing the grism angle was therefore investigated by rotating the grism assembly away from the beam axis. The initial position was with the grism aligned along the beam axis (rotation $=0^{\circ}$ ) and the rotation was incrementally increased up to $5^{\circ}$. The rotation axis was vertical, parallel to the VPH grating lines, and coincident with centre of the first surface of the leading prism. The relative change in emission line intensities from a $\mathrm{Kr}$ arc source are shown in Figure 5 suggesting that a selectable grism angle would be of advantage. The degree of tilt is small enough that no motion of the camera and detector system was needed to compensate for the slight motion of the spectrum that results.

The instrument will therefore operate in one of two selectable wavelength optimised modes. The normal mode centered at $6000 \AA$ and a blue optimised mode, peaking at $5000 \AA$. The rotation angle will be set using a simple pneumatically actuated mechanism. 


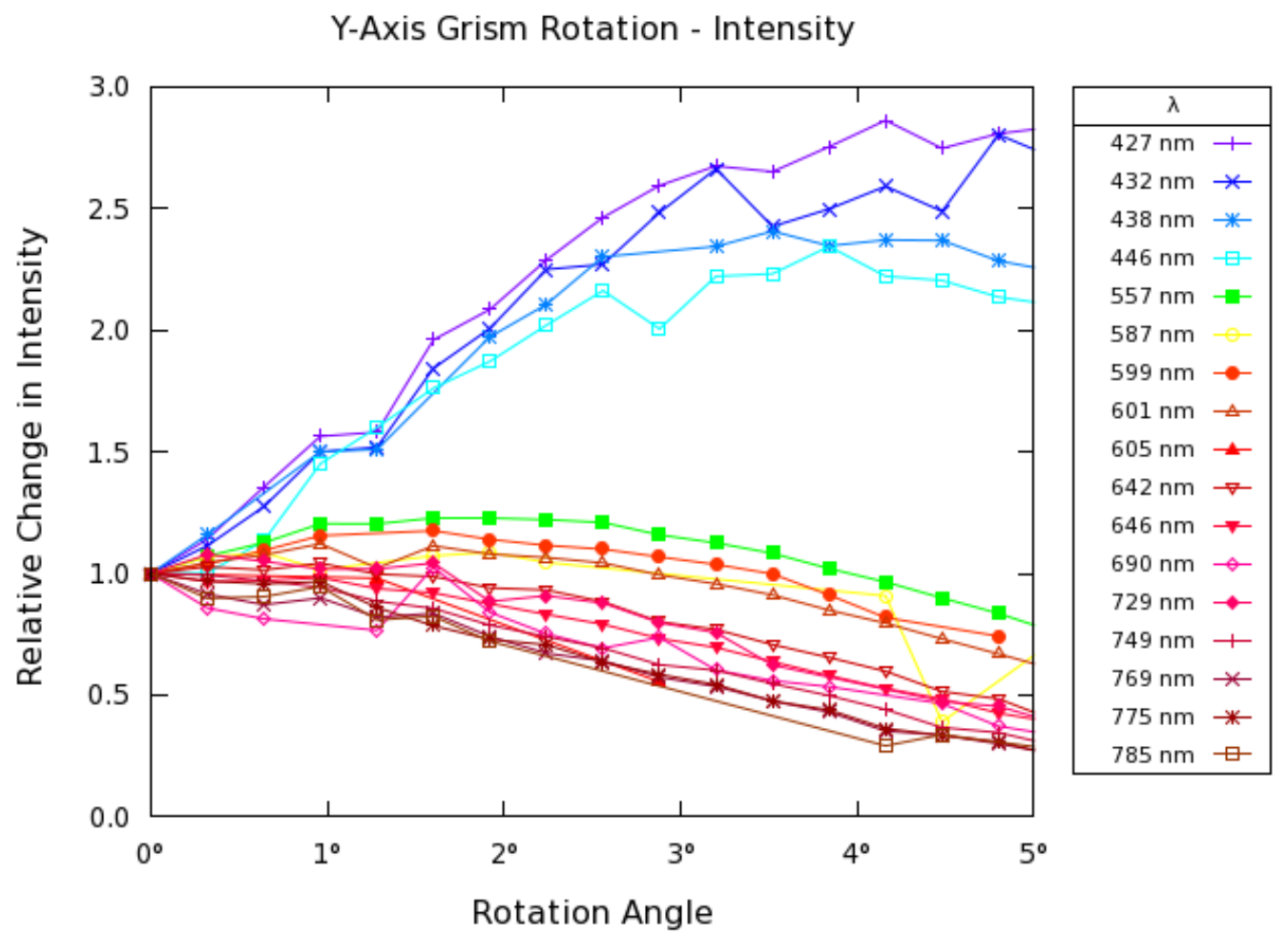

Figure 5. The relative change in detected intensities as a function of grism rotation angle for a krypton arc. The prototype instrument was optimised for green, $\sim 550 \mathrm{~nm}$, which peaked at $\sim 1.75^{\circ}$. Increasing the angle showed that the blue 446 $\mathrm{nm}$ line peaked at $\sim 3.5^{\circ}$, more than doubling in intensity.

\section{PREDICTED SENSITIVITY}

The photon efficiency $E_{\text {total }}$ is the proportion of photons arriving at the telescope aperture that can be detected by the camera. It is the product of the camera quantum efficiency (QE), and the throughput of each individual element along the optical path, assuming ideal minimum losses ...

$$
E_{\text {total }}=Q E \times E_{\text {pri }} \times E_{\text {sec }} \times E_{\text {sideport }} \times \ldots
$$

In addition to the primary and secondary mirror reflection losses, the instrument will be mounted on a side-port so will suffer additional attenuation from the pick-off mirror. Table 1 shows the optical elements and individual throughput at $6000 \AA$. The predicted throughputs over the full instrument range $4000-8000 \AA$ are shown in Figure 6.

Shown in Figure 7 are predicted signal-to-noise ratios for a selection of object magnitudes. Objects with magnitude $M_{v}=20$, will required extended exposures $\sim 500 \mathrm{~s}$.

\section{CALIBRATION}

\subsection{Wavelength Calibration Lamp}

For all spectrographs, calibration essential to provide a mapping between pixel position along the dispersion axis of spectrum image and the corresponding wavelength. A nobel gas arc lamp provides a suitably stable source with accurately identifiable lines. Three arc lamps were tested (krypton, neon and xenon) made by LOT-Oriel( Figure 8$)$. The limited range of Ne lines $(\sim 5800-7000 \AA)$ made it unsuitable as a source. Krypton had the widest range but has a bright $7602 \AA$ line which saturated the CCD when longer exposures were taken to detect weak lines. All the arc spectra exhibited a gap around $5300 \AA$. To reduce the gap and extend the ranges of $\mathrm{Kr}$ and 


\begin{tabular}{|l|c|c|c|}
\hline \multicolumn{1}{|c|}{ Element } & $\begin{array}{c}\mathrm{E} \\
\text { per Element }\end{array}$ & $\begin{array}{c}\text { Elements/ } \\
\text { Surfaces }\end{array}$ & Total \\
\hline Liverpool Telescope $\left(E_{\text {pri }} \times E_{\text {sec }} \times E_{\text {sideport }}\right)$ & 0.810 & 1 & 0.810 \\
Slit & 0.970 & 1 & 0.970 \\
Field lens - VIS AR coating & 0.995 & 2 & 0.990 \\
Long pass filter - VIS AR coating & 0.910 & 1 & 0.910 \\
Collimator lens - VIS AR coating & 0.995 & 2 & 0.990 \\
Prism - VIS AR coating & 0.995 & 4 & 0.980 \\
Grating substrate - AR coating & 0.995 & 4 & 0.980 \\
VPH grating & 0.850 & 1 & 0.850 \\
Camera lens - AF Nikkor 85mm f/1.8D & 0.940 & 1 & 0.940 \\
CCD QE - Andor iDus 420-BV & 0.850 & 1 & 0.850 \\
\hline Total Efficiency $\left(E_{\text {total }}\right)$ & \multicolumn{3}{|c|}{0.457} \\
\hline
\end{tabular}

Table 1. Estimated Photon Efficiency at $\lambda=6000 \AA$. Slit losses have been calculated assuming a 2.16 arcsecond slit and modelling the atmospheric seeing as a Gaussian profile with FWHM $=1.0$ arcseconds.

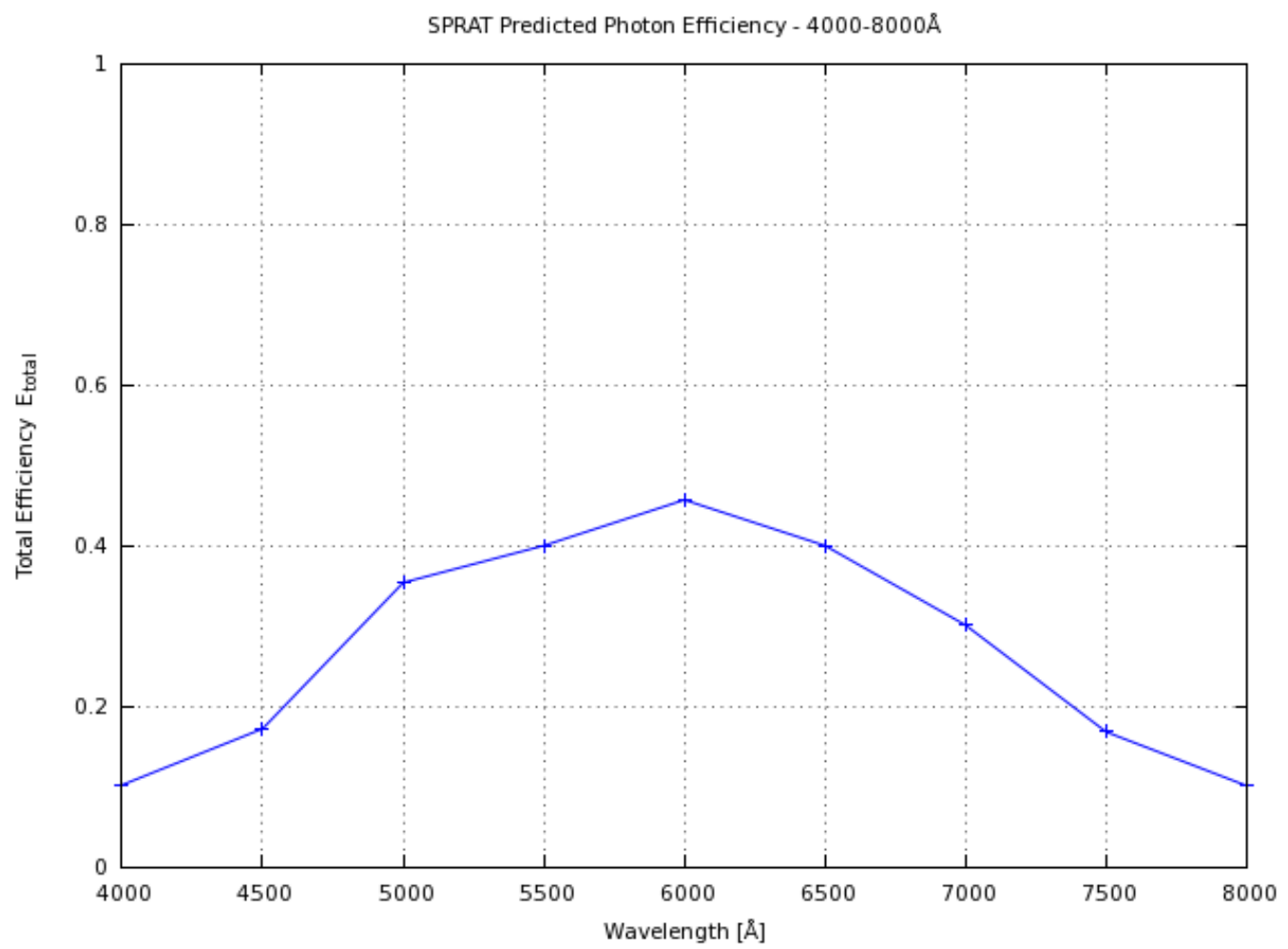

Figure 6. The total photon efficiency is predicted to peak at the central wavelength of $6000 \AA$ with the beam normally incident to the prism/grating assembly

$\mathrm{Xe}$, the manufacturer's standard list was augmented with lines from the NIST reference web-site and the arc calibration was repeated. The updated results, Figure 9, show xenon's greater range and lacks the bright line feature of krypton. Xenon was therefore selected as the arc calibration source.

\subsection{Flat field lamp}

The instrument incorporates an internal flat light source for characterising the response of the CCD pixels. With the slit and grating removed this will provide data on small (pixel) scale sensitivity variations. In addition with the slit and grating in place it will provide data on the larger scale system spectral response assuming it is a 

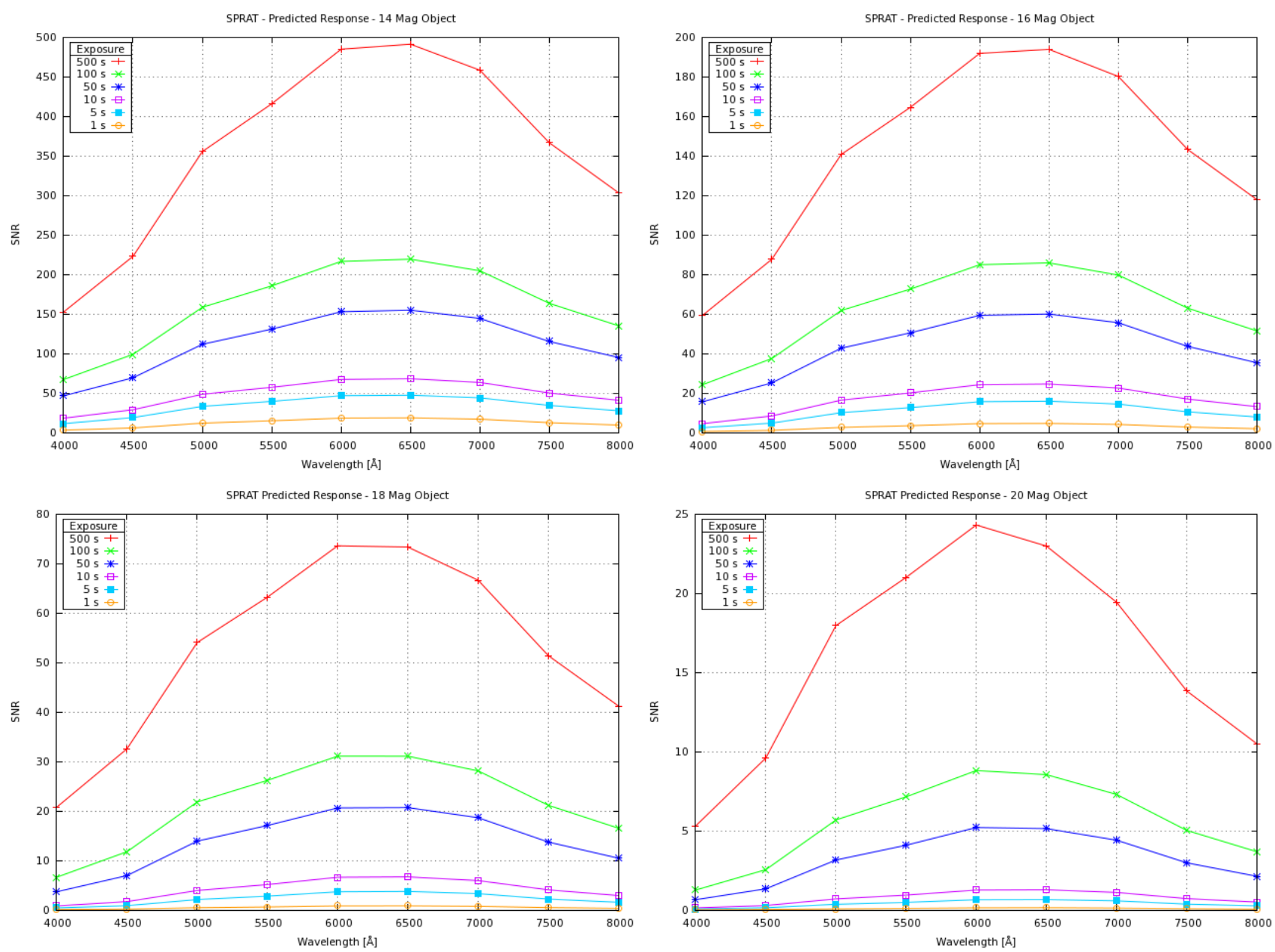

Figure 7. Predicted SNRs at selected exposures for object of magnitude $M=14,16,18 \& 20$.

continuum source. Sky flats will be used to provide data on the slit profile. The flat source is introduced in to the beam ahead of the slit via a movable mirror and light pipe. Several white LEDs and low voltage incandescent lamps were evaluated as suitable flat light sources. The LEDs showed a discontinuous spectrum and continue to phosphoresce, emitting light after they were turned off, so were discounted. The spectra for a variety of $\sim 12 \mathrm{~V}$ incandescent lamps were investigated (Figure 10). The clear Bosch434 tungsten halide lamp at 13V showed the best blue response. The effective colour temperature of a lamp was increased by suppressing long wavelengths with a blue filter to "flatten" the spectrum. Filters are often manufactured using a combination of dyes and produce a modulated response. Figure 11 presents the 4 least modulated spectra from the Roscolux set of blue filter gels. Roscolux $32161 / 8^{\text {th }}$ Blue was chosen as the companion filter to go with the Bosch434 flat lamp.

\section{LABORATORY TESTING}

An experimental layout of the spectrograph using the actual optical components but temporary mechanical mountings was setup on an optical table. This setup was used to carry out the tests on throughput described above, and also provided a means of developing setup procedures and testing system stability.

The optics were centered on a principle axis defined using a $532 \mathrm{~nm}$ green laser. The axis was aligned to $<0.2^{\circ}$ using pin-hole templates separated by $280 \mathrm{~mm}$. The lateral alignment of the optical components was checked relative to this axis. Angular alignment was done using a simple auto-collimation method of reflecting the laser light from the centre of an optical component back to the laser source. 


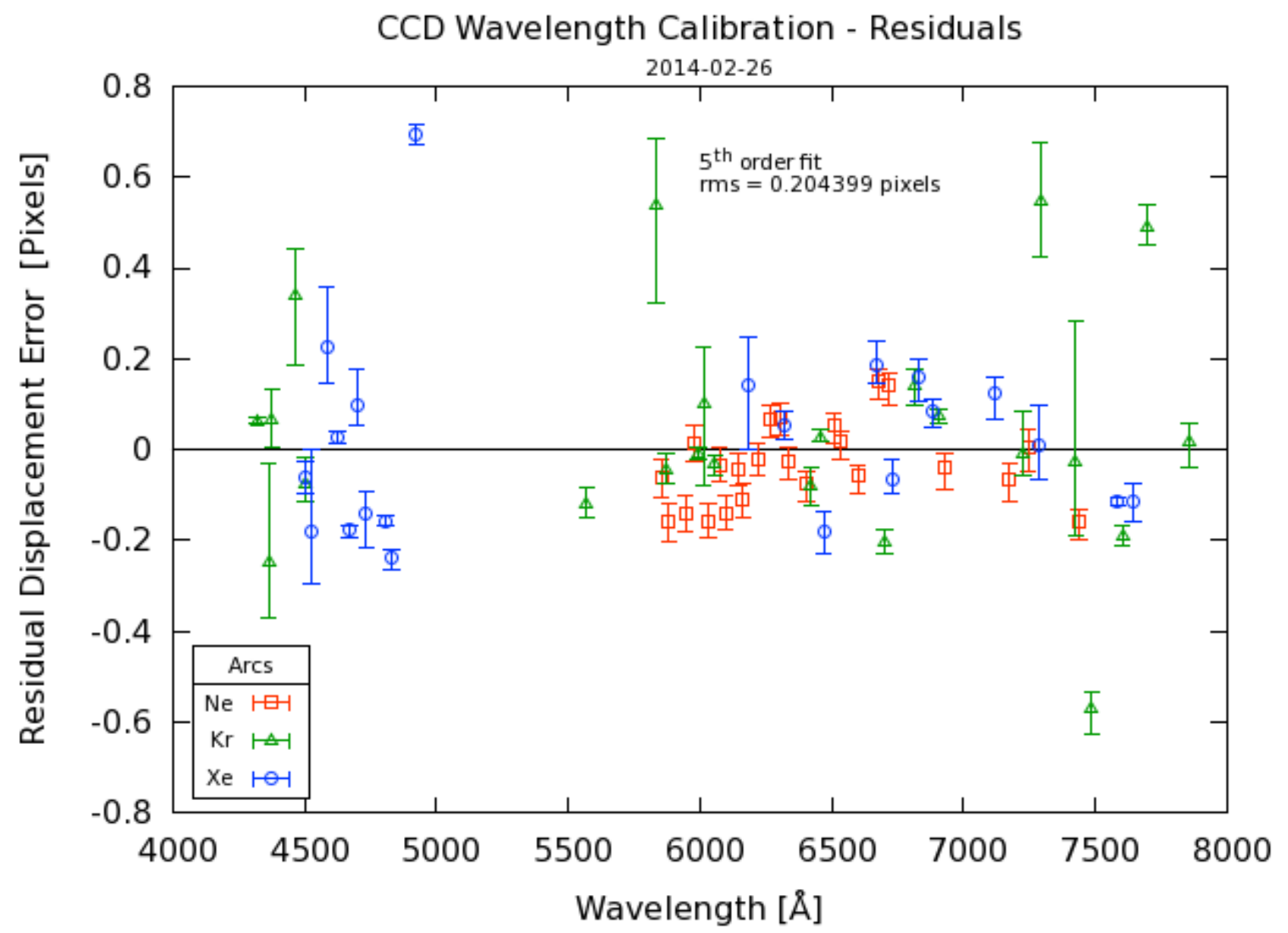

Figure 8. Initial calibration arc lamp evaluation showed Ne as unsuitable because of its limited range of lines in the visible. Kr provided the widest range but produces a bright line at $7602 \AA$. All arcs showed a gap at centered around $5300 \AA$.

The grism rotation tests using the a $\mathrm{Kr}$ arc source (which has a very bright $7602 \AA$ line) clearly showed several ghost lines and other reflection artifacts. To identify the origin of the ghosting the optical axis alignment laser and Kr lamp were used as a bright sources together with a white screen/surface to detect reflections.

The grism body was machined from acetal polymer giving the entrance and exit ports a smooth reflective surface. Placing a white paper on these surfaces and illuminating with the optical axis alignment laser showed secondary reflection from within the grism impinging on the port sides. In the final instrument the ports will therefore be redesigned to reduce the length from $20 \mathrm{~mm}$ to $9.2 \mathrm{~mm}$ and a bevel added to allow light to escape to the instrument enclosure and be absorbed by baffles. The acetal surface will also be bead blasted to reduce specular reflection. In the laboratory setup installing non-reflective lining material successfully removed a large diffuse feature at the red end of the spectrum.

Optical elements were sequentially removed or rotated to detect any corresponding change in ghosting. It was identified that the long-pass filter placed immediately ahead the grism was re-reflecting light coming from the front/rear surfaces of the grating. This was eliminated by moving the long pass filter to immediately behind the field lens. Internal baffling will be added to absorb the redirected reflections.

To detect ghosting originating from optical elements within the grism, deformable elastomeric rings were placed between the grating and prisms to mechanically decouple the elements. The screws holding the prism mount were then adjusted to selectively move a single prism. Reflections were observed on screens placed ahead 


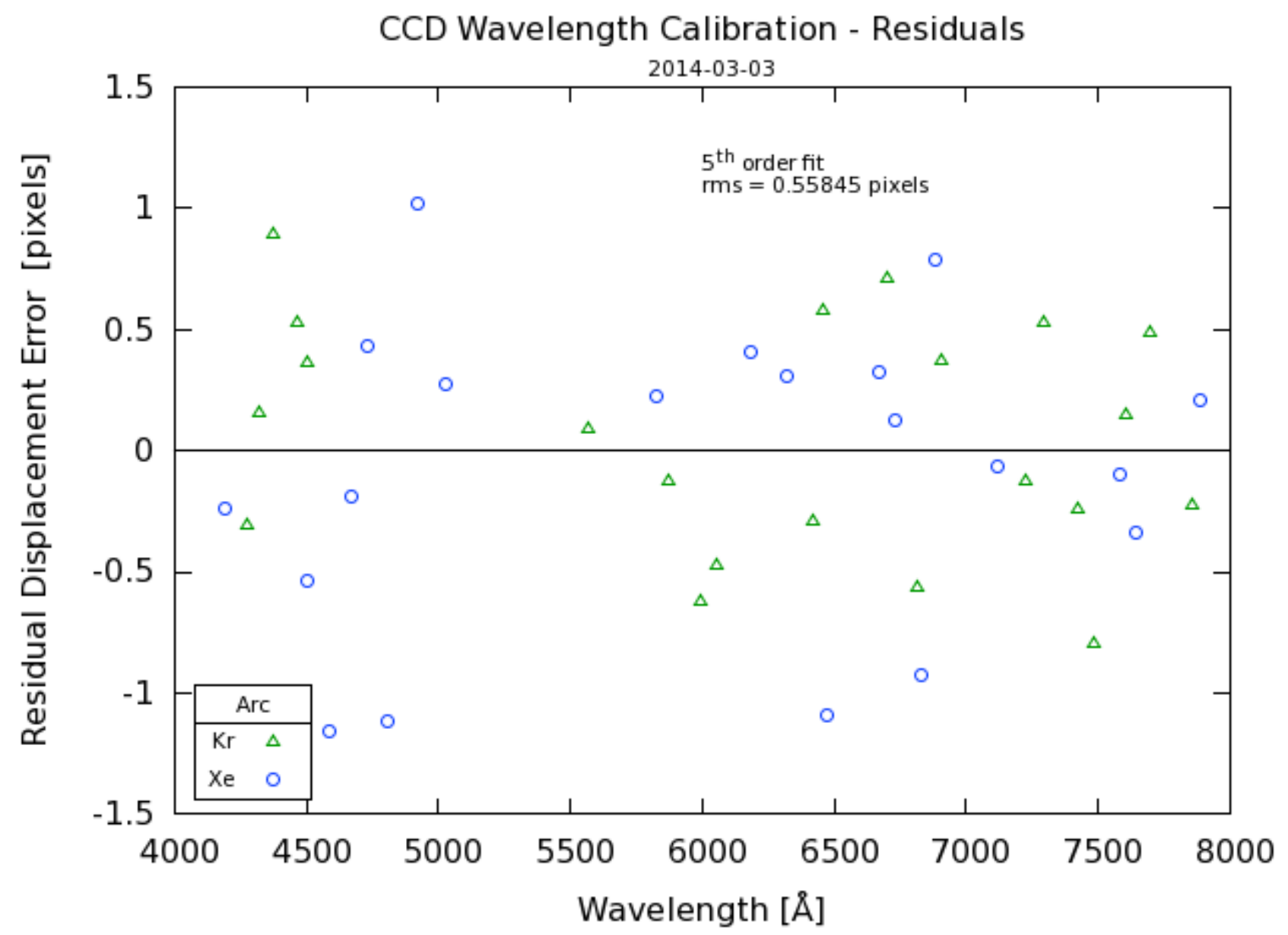

Figure 9. Calibration arc evaluation augmented with lines from NIST. Xenon now has the widest overall range and a reduced $\sim 5300 \AA$ gap so was selected as calibration source.

and behind the assembly. The primary ghosting source was established to come from the grating glass and was reduced by tilting it down $\sim 1^{\circ}$ in the direction of the optical axis.

For very bright sources causing pixel saturation, 2 residual ghost lines remained but were determined to be $>2$ orders of magnitude fainter than the saturated line and ignorable. It was noted these ghost lines are displaced vertically from the main spectrum. As an additional ghost line detection method the slit was masked so that the spectrum occupied a central band $\sim 200$ vertical pixels in height. Ghost lines are easily identified if they extend beyond the upper or lower edges of a spectrum, into this masked-off region.

Focus tests showed a noticeable slit image defocus for changes of $\pm 0.028 \mathrm{~mm}$ in the slit to collimator distance, $\sim 150 \mathrm{~mm}$. The operating temperature for the instrument is -5 to $20^{\circ} \mathrm{C}$. Over this range only a few conventional steels provide an acceptably low expansion, e.g. austenitic steel produces $\pm 0.025 \mathrm{~mm}$ which is just acceptable. Invar was therefore selected as the baseplate material for mounting the optical mounts between slit and collimator with a predicted expansion of $\pm 0.0025 \mathrm{~mm}$. Exiting the collimator the beam is parallel so distance changes will not effect the focus. 
Flatlight Source Comparison

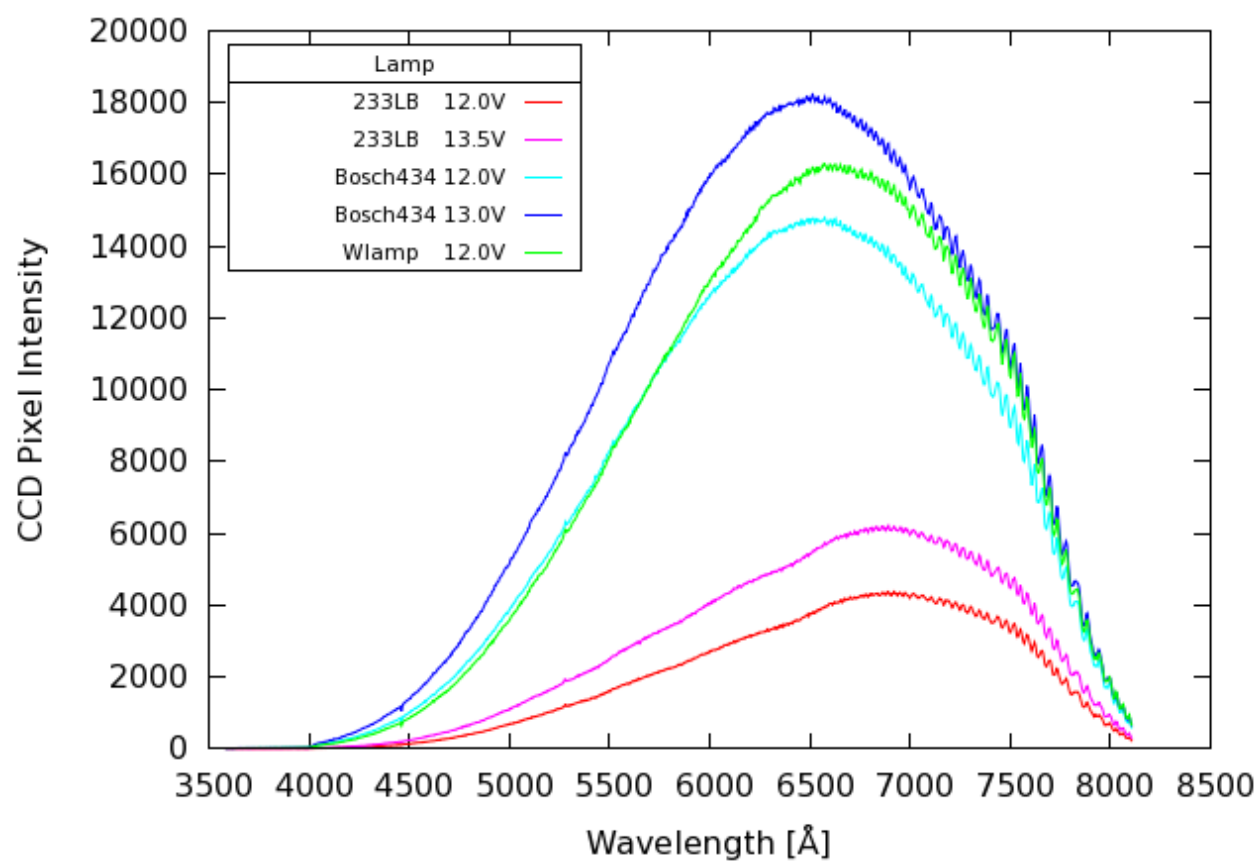

Figure 10. Wlamp is a generic $12 \mathrm{~V}$ tungsten torch bulb. Two automotive lamps were evaluated. 233LB is tungsten with blue filter and Bosch434 a clear tungsten halide. Automotive lamps are designed to operate at $12.6 \mathrm{~V}$ so were operated at $12.0 \mathrm{~V}$ and at a small over voltage for comparison.

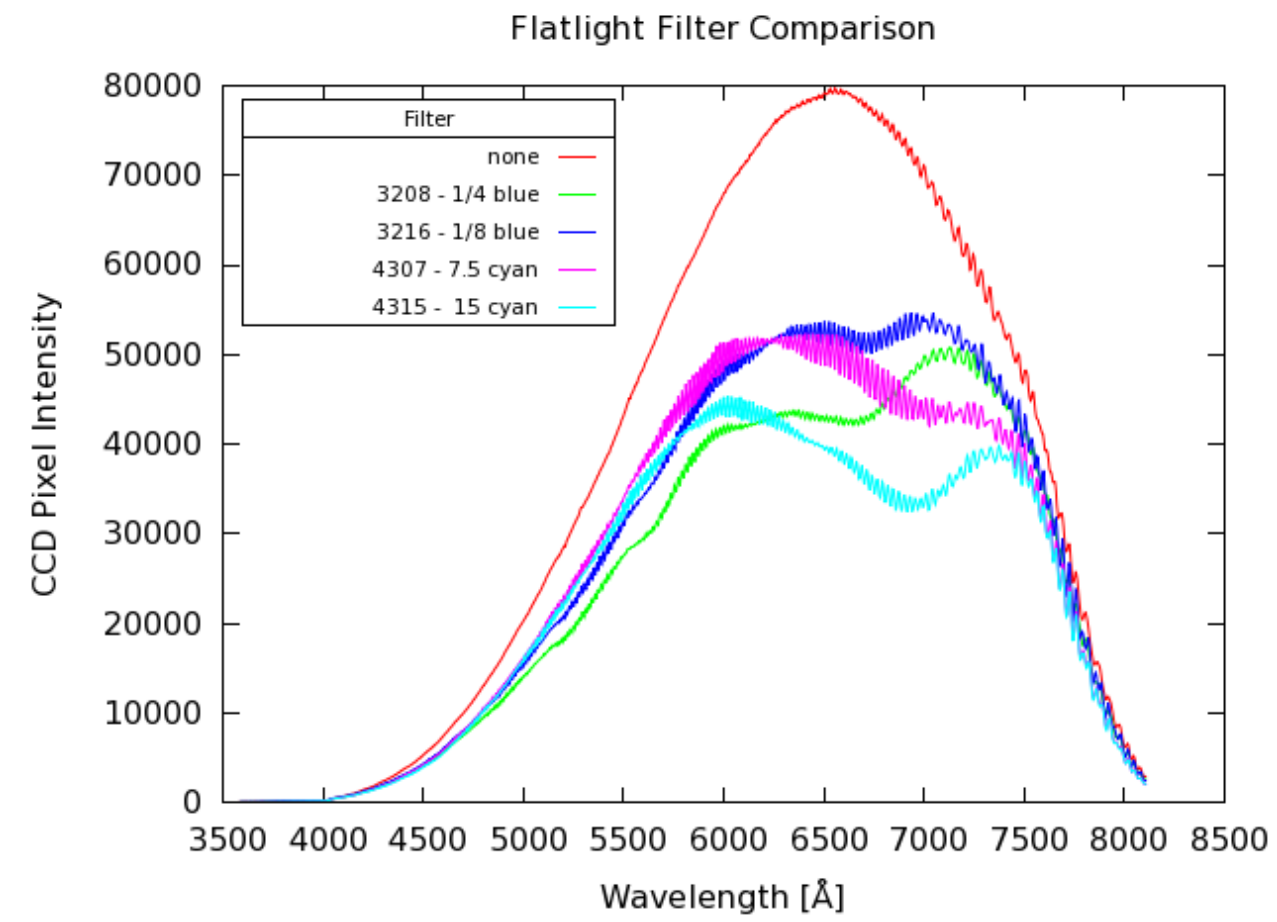

Figure 11. The effect of blue gel filters on the spectrum of the Bosch434 tungsten halide bulb was evaluated. Roscolux 3216 showed the least modulation and increased the relative intensity in the blue. 


\section{MECHANICAL DESIGN}

\subsection{Materials}

The structure of the instrument is predominantly aluminium due to its reduced cost, easy machinability and relatively light weight. As accurate distances between the optics must be maintained, the design employs an internal pseudo optical bench made from Invar because of its substantially lower coefficient of thermal expansion. One end of the Invar plate is pinned to the instrument structure at the slit position, (co-incident with the telescope focus plane). The other end 'floats', allowing the rest of the instrument to expand at a higher rate without deforming the structure. To compensate for changes in the gravity vector, the floating portion of the Invar is constrained using Belleville washers stacked back-to-back to apply a relatively high clamping force ( $240 \mathrm{~N})$ within a small space envelope.

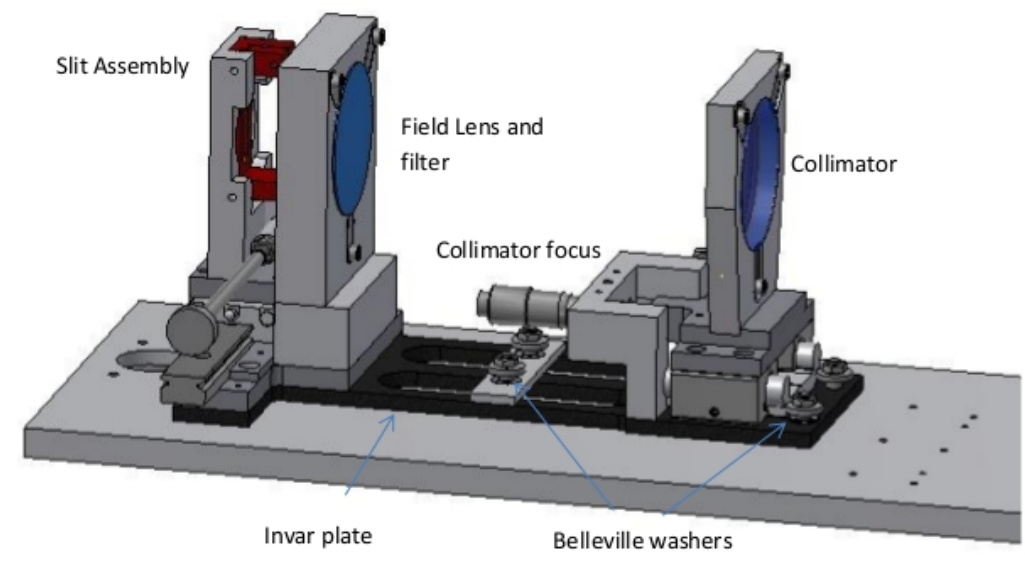

Figure 12. Pseudo optical bench sub-assembly.

\subsection{Movable Components}

At commissioning the collimator will require a one-off adjustment of the focus. The numbered components are movable along re-circulating ball linear guides and deployed using SMC compact pneumatic cylinders.

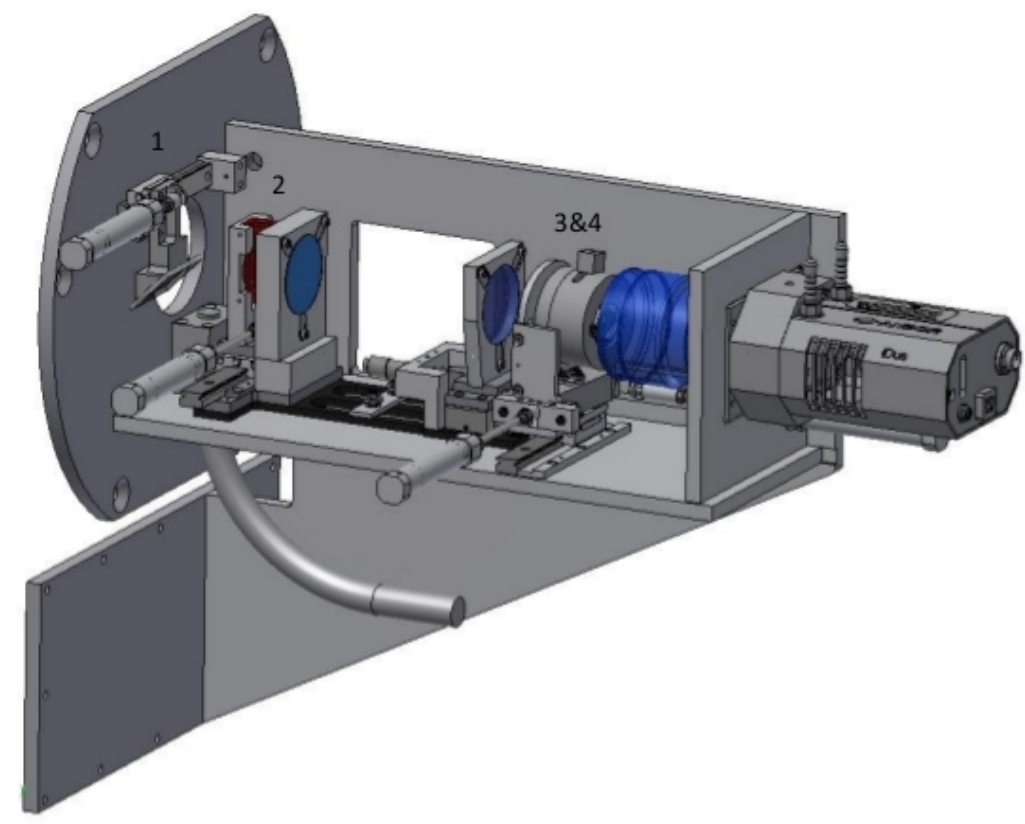

Figure 13. 1. Calibration mirror deployment, 2. Slit deployment, 3\&4. Grism and rotation angle stop deployment 


\subsection{Flexure}

Figure 14 (telescope at zenith, instrument upright and horizontal) and Figure 15 (telescope at horizon, instrument on its side and horizontal) are FEA models of the instrument mounting and enclosure showing deflection. The yellow arrow indicates the direction of the gravity vector. The colour regions indicate magnitude of deflection and are drawn with an exaggerated displacement from the un-deflected instrument (outlined in black). The worst case mechanical flexure is an order of magnitude less than $0.023 \mathrm{~mm}$ needed for a $\frac{1}{2}$ pixel shift. To further minimise the flexure both the upper and lower ports of the LT A\&G box are utilised to provide an angled brace.

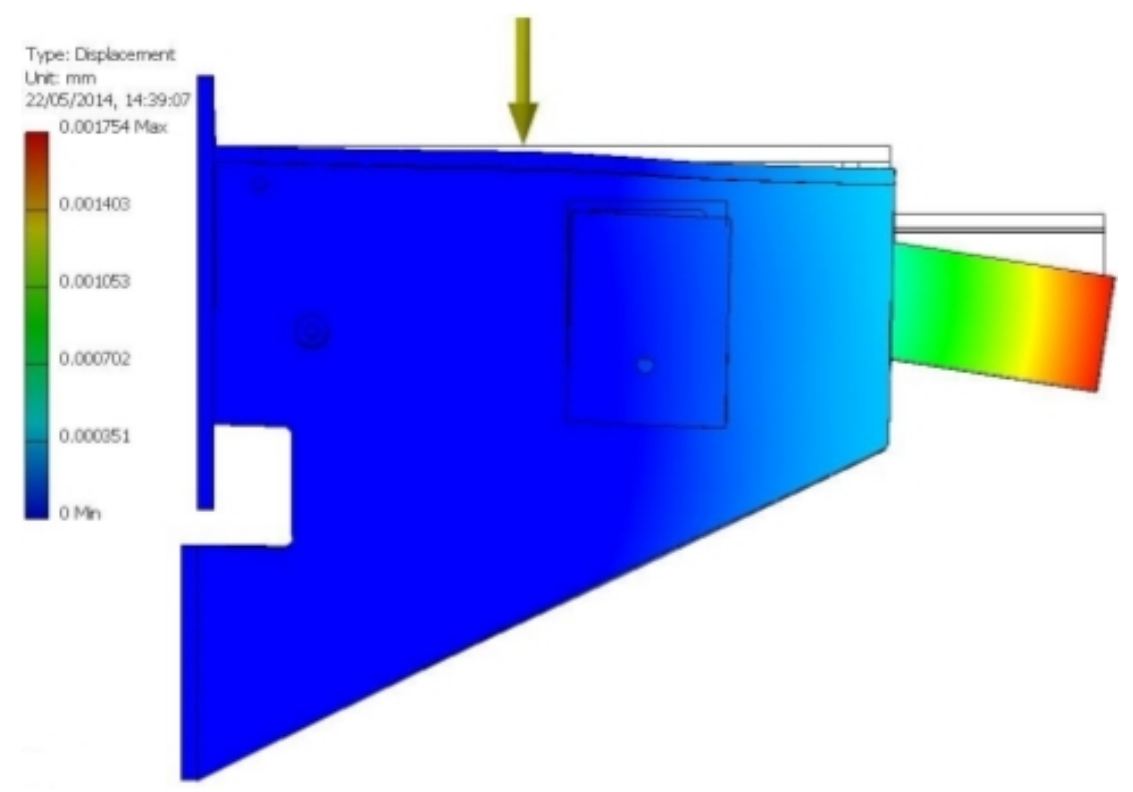

Figure 14. Telescope at zenith, A\&G box rotated with instrument upright and horizontal, the maximum predicted deflection at the rear of the camera is $0.0017 \mathrm{~mm}$.

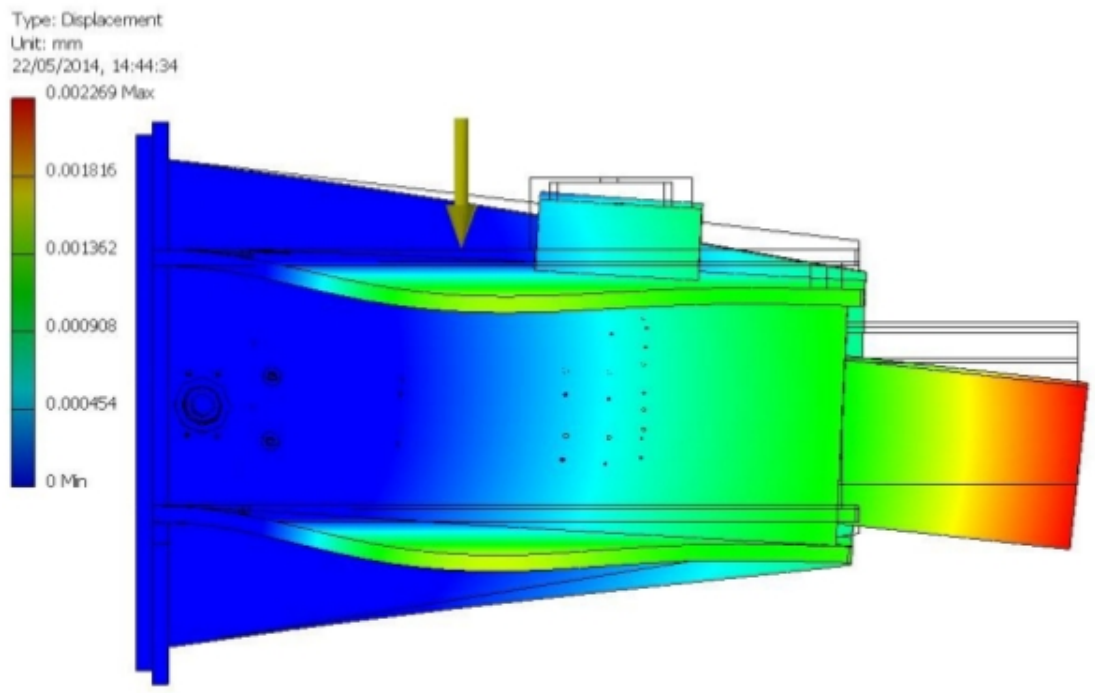

Figure 15. Telescope at horizon (worst case and not actually possible as min. altitude angle is $20^{\circ}$ ), A\&G box rotated with instrument on its side and horizontal, the maximum predicted displacement at the rear of the camera is $0.0022 \mathrm{~mm}$. 


\subsection{Plan View of Instrument}

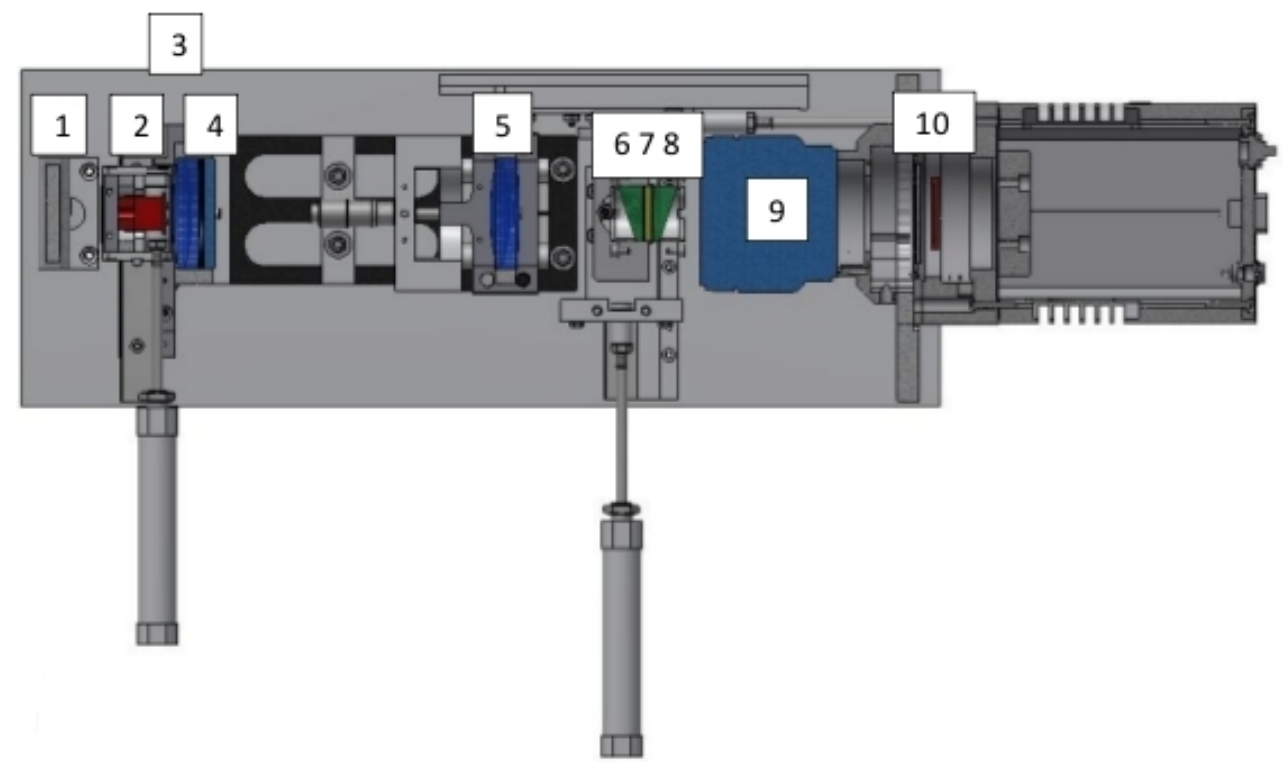

Figure 16. A plan view of the instrument identifying the optical elements. 1. Arc/Flat Calibration Angled Mirror (dark grey), 2. Slit (red), 3. Field Lens (blue), 4. Long-pass Filter (cyan), 5. Collimator Lens (blue), 6. $1^{\text {st }}$ Prism (green), 7. VPH Grating (yellow), 8. $2^{\text {nd }}$ Prism (green), 9. Camera Lens (cyan), 10. CCD (dark red)

\section{CONCLUSIONS}

We have described the design and laboratory testing of a new, low resolution spectrograph designed for robotic operation. By using a VPH/prism combination we have achieved a straight optical path, allowing the spectrograph detector to be used for image acquisition with the slit and grating moved out of the beam. Our experiments with the telescope on sky predict that by an iterative process of imaging and telescope offsets we should be able to position the target such that once the slit is deployed into the beam it will lie within it. A small "peak-up" procedure may then be necessary to centre the object within the slit.

The design has been optimized for as high a throughput over as wide a wavelength range as possible. Experiments with the VPH grating showed that the change in peak throughput wavelength with input angle are sufficiently large that we have had to implement a two position, grating tilt mechanism to allow observers to tune the instrument performance to their science goals.

The instrument is now in manufacture, and will be deployed to the telescope over Summer 2014. Following commissioning, an automated data reduction pipeline ${ }^{18}$ will be developed.

\section{ACKNOWLEDGMENTS}

The Liverpool Telescope is operated on the island of La Palma by Liverpool John Moores University in the Spanish Observatorio del Roque de los Muchachos of the Instituto de Astrofisica de Canarias with financial support from the UK Science and Technology Facilities Council and the EU FP7.2 OPTICON project.

\section{REFERENCES}

[1] Steele, I. A., Smith, R. J., Rees, P. C., Baker, I. P., Bates, S. D., Bode, M. F., Bowman, M. K., Carter, D., Etherton, J., Ford, M. J., Fraser, S. N., Gomboc, A., Lett, R. D. J., Mansfield, A. G., Marchant, J. M., Medrano-Cerda, G. A., Mottram, C. J., Raback, D., Scott, A. B., Tomlinson, M. D., and Zamanov, R., "The Liverpool Telescope: performance and first results," in [Ground-based Telescopes], Oschmann, Jr., J. M., ed., Society of Photo-Optical Instrumentation Engineers (SPIE) Conference Series 5489, 679-692 (Oct. 2004). 
[2] Steele, I. A., Bates, S. D., Carter, D., Clarke, D., Gomboc, A., Guidorzi, C., Melandri, A., Monfardini, A., Mottram, C. J., Mundell, C. G., Scott, A. B., Smith, R. J., and Swindlehurst, J., "RINGO: a novel ring polarimeter for rapid GRB followup," in [Society of Photo-Optical Instrumentation Engineers (SPIE) Conference Series], Society of Photo-Optical Instrumentation Engineers (SPIE) Conference Series 6269 (July 2006).

[3] Arnold, D. M., Steele, I. A., Bates, S. D., Mottram, C. J., and Smith, R. J., "RINGO3: a multi-colour fast response polarimeter," in [Society of Photo-Optical Instrumentation Engineers (SPIE) Conference Series], Society of Photo-Optical Instrumentation Engineers (SPIE) Conference Series 8446 (Sept. 2012).

[4] Mundell, C. G., Kopač, D., Arnold, D. M., Steele, I. A., Gomboc, A., Kobayashi, S., Harrison, R. M., Smith, R. J., Guidorzi, C., Virgili, F. J., Melandri, A., and Japelj, J., "Highly polarized light from stable ordered magnetic fields in GRB120308A," Nature 504, 119-121 (Dec. 2013).

[5] Mottram, C. J., Steele, I. A., and Morales, L., "Design of low cost and reliable instrumentation for robotic telescopes," in [Ground-based Instrumentation for Astronomy], Moorwood, A. F. M. and Iye, M., eds., Society of Photo-Optical Instrumentation Engineers (SPIE) Conference Series 5492, 677-688 (Sept. 2004).

[6] Morales-Rueda, L., Carter, D., Steele, I. A., Charles, P. A., and Worswick, S., "The Liverpool Telescope Spectrograph: FRODOSpec," Astronomische Nachrichten 325, 215-215 (Mar. 2004).

[7] Shalyapin, V. N. and Goicoechea, L. J., "Spectra of faint sources in crowded fields with FRODOSpec on the Liverpool Robotic Telescope," Astronomische Nachrichten 335, 428 (2014).

[8] Jewitt, D., "Project Pan-STARRS and the Outer Solar System," Earth Moon and Planets 92, 465-476 (June 2003).

[9] Law, N. M., Kulkarni, S. R., Dekany, R. G., Ofek, E. O., Quimby, R. M., Nugent, P. E., Surace, J., Grillmair, C. C., Bloom, J. S., Kasliwal, M. M., Bildsten, L., Brown, T., Cenko, S. B., Ciardi, D., Croner, E., Djorgovski, S. G., van Eyken, J., Filippenko, A. V., Fox, D. B., Gal-Yam, A., Hale, D., Hamam, N., Helou, G., Henning, J., Howell, D. A., Jacobsen, J., Laher, R., Mattingly, S., McKenna, D., Pickles, A., Poznanski, D., Rahmer, G., Rau, A., Rosing, W., Shara, M., Smith, R., Starr, D., Sullivan, M., Velur, V., Walters, R., and Zolkower, J., "The Palomar Transient Factory: System Overview, Performance, and First Results," PASP 121, 1395-1408 (Dec. 2009).

[10] Prusti, T., "The promises of Gaia," Astronomische Nachrichten 333, 453 (June 2012).

[11] Bloom, J. S., Richards, J. W., Nugent, P. E., Quimby, R. M., Kasliwal, M. M., Starr, D. L., Poznanski, D., Ofek, E. O., Cenko, S. B., Butler, N. R., Kulkarni, S. R., Gal-Yam, A., and Law, N., "Automating Discovery and Classification of Transients and Variable Stars in the Synoptic Survey Era," PASP 124, 1175-1196 (Nov. 2012).

[12] Blagorodnova, N., Koposov, S. E., Wyrzykowski, b., Irwin, M., and Walton, N. A., "GS-TEC: the Gaia Spectrophotometry Transient Events Classifier," ArXiv e-prints (Apr. 2014).

[13] Ngeow, C.-C., Konidaris, N., Quimby, R., Ritter, A., Rudy, A. R., Lin, E., and Ben-Ami, S., "The SED Machine: A Spectrograph to Efficiently Classify Transient Events Discovered by PTF," in [IAU Symposium], Zhang, C. M., Belloni, T., Méndez, M., and Zhang, S. N., eds., IAU Symposium 290, 281-282 (Feb. 2013).

[14] Mink, D. J., "WCSTools: Image World Coordinate System Utilities," in [Astronomical Data Analysis Software and Systems VI], Hunt, G. and Payne, H., eds., Astronomical Society of the Pacific Conference Series 125, 249 (1997).

[15] Hill, G. J., Wolf, M. J., Tufts, J. R., and Smith, E. C., "Volume phase holographic (VPH) grisms for infrared and optical spectrographs," in [Specialized Optical Developments in Astronomy], Atad-Ettedgui, E. and D'Odorico, S., eds., Society of Photo-Optical Instrumentation Engineers (SPIE) Conference Series 4842, 1-9 (Feb. 2003).

[16] Harmer, C. F. W., "Pupil Imagery in Astronomical Spectrographs," Monthly Notes of the Royal Astronomical Society 167, 311-318 (May 1974).

[17] Baldry, I. K., Bland-Hawthorn, J., and Robertson, J. G., "Volume Phase Holographic Gratings: Polarization Properties and Diffraction Efficiency," PASP 116, 403-414 (May 2004).

[18] Barnsley, R. M., Smith, R. J., and Steele, I. A., "A fully automated data reduction pipeline for the FRODOSpec integral field spectrograph," Astronomische Nachrichten 333, 101-117 (Feb. 2012). 